\title{
A Multiple-Derivative and Multiple-Delay Paradigm for Decentralized Controller Design: Uniform-Rank Systems
}

Yan Wan

Sandip Roy

\section{INTRODUCTION}

Decentralized feedback systems have long been of interest to the controls community [2], [3], [20]. In recent years, research in decentralized control has been re-invigorated by interest in such applications as cooperative control of autonomous vehicle teams, data fusion in sensor networks, air traffic management, and virus-spreading control, among many others, see the overviews [15]-[18] and also e.g. the articles [6], [19], [23]. These numerous network-control applications are widely varied: they have vastly different scales, may consist of autonomous-but-sensing agents (like vehicle teams) or may be hardwired (like electric power systems), and require a wide array of control and/or algorithmic capabilities. What these varying applications make clear, however, is that new tools for designing decentralized controllers are badly needed: ones that permit highly limited components subject to delay and variation to complete intricate tasks by exploiting the network's topological structure. As the need for topology-exploiting decentralized controllers has become clear, efforts to understand the role of a network's topology in its dynamics upon control have been initiated, but the core design problem remains to be addressed.

Given the long history of decentralized control, the reader may well wonder why new techniques are still needed for decentralized controller design. Historically, decentralized controller design has focused on making network components individually impervious to network interactions [2], or has concentrated the complexity and actuation at one component in the network [3]. However, the recent study of sensing-agent networks [17], as well as certain infrastructure networks such as air traffic management systems [4] and electric power systems [5], makes clear that a single agent cannot provide the actuation or complexity required to control the whole network, and further the controllers must exploit the network topology to cooperatively achieve performance requirements. This article pursues design of such topology-based and cooperative decentralized controllers.

For a clearer motivation, let us trace the decentralized controls literature. We stress that the bulk of the traditional decentralized control theory views the network as a disturbance that must be dominated by the local dynamics [2], and hence does not permit design or analysis of controllers that exploit the network topology. The seminal work of Wang and Davison [20] does make the role played by the network explicit, in that it gives necessary and sufficient conditions for stabilization of decentralized systems based on fixed modes (see also, e.g., [3], [7], [8]). Their methodology is very

\author{
Ali Saberi \\ Anton Stoorvogel
}

much applicable to modern networks, and we have used it to address the foundational problem of determining whether a sensing-agent network can be stabilized [23]. Unfortunately, Wang and Davison's perturbation-based approach does not permit constructive design of practical high-performance or even stabilizing controllers. While several works have extended [20] toward allowing eigenvalue placement (and hence high performance) in addition to stabilization, these approaches essentially concentrate the complexity and extent of actuation/observation at a single agent, and hence also are unsuitable for our applications [3]. For these reasons, new tools for decentralized controller design are badly needed.

Recognizing the need for new decentralized controller designs, our group over the last five years has addressed a family of canonical dynamical network design tasks (e.g., [19], [24]-[26]). These include static decentralized controller design and network graph edge-design for stabilization, performance optimization, and performance shaping; both partial and full network design problems have been addressed, and design in the presence of constraints has been attempted. Through addressing these various canonical design problems, we have developed a suite of tools for designing static decentralized controllers that exploit a network's topological structure, based on optimization machinery, time-scale concepts, and algebraic graph theory. We have shown that these network-theoretic static controller designs have wide application, in such diverse fields as air traffic management, virus-spreading control, and vehicle-team coordination. Yet, since the static controllers typically achieve good performance only when the network's internal variables (states) are all directly measured among the channels, these static controller designs are necessarily limited to systems with simple network components and sufficient design freedoms. In fact, many modern networks have much more intricate (and significantly hidden) dynamics, and so we are strongly motivated to pursue the design of dynamical decentralized controllers. Here, we introduce a philosophy for dynamical controller design that builds on our static control efforts, and begins to address the decentralized controller design problem for a wide class of models and applications.

Specifically, in this article we introduce a promising methodology for designing stabilizing and high-performance yet practical topology-exploiting dynamic controllers for LTI decentralized systems, that is fundamentally based on 1) envisioning a control architecture with direct local feedback of multiple derivatives of the observation and 2) using multilead-compensator and multiple-delay control schemes to implement these multiple-derivative controllers. We show that 
this new signal-based methodology is capable of addressing many of the complexities that are common to modern decentralized systems, including very general observation topologies, saturation nonlinearities, and inherent network delays. In this installment, we motivate and introduce the design methodology for the wide class of (centrally) squareinvertible uniform-rank plants. We focus on this class for three reasons: 1) because we have encountered decentralized plants with this structure in numerous infrastructural and sensing-agent networks, 2) to give an uncluttered presentation of the intricate design methodology, and 3) as a steppingstone toward a design for general LTI decentralized plants.

Before pursuing technical details, let us motivate the two core aspects of our design methodology: namely, the multiple-derivative control paradigm and the leadcompensator- and delay- based implementations. First, let us consider the multiple-derivative control paradigm. Broadly, in LTI systems, the derivatives of observations provide information about the entire system's state, and so permit control. Classically, this inference of the system state is achieved through use of an observer; however, this observer-based paradigm fails in a decentralized system where the full output is not available anywhere, and so we are instead motivated to envision state computation through direct use of output derivatives. With the motivation, we postulate a control architecture in which output derivatives are used explicitly in feedback. This use of derivatives of the local observations in feedback provides the channels (upon closing the loop) with statistics of the part of the global state associated with the infinite-zero structure, and so facilitates control. A core novelty in our architecture, as compared to those envisioned for centralized control problems, is in the highgain feedback of output derivatives equal to the (common) order of the infinite zeros in the uniform-rank model (i.e. the depth of the integrator chain, or "relative degree" of the model). This control architecture envisions use of one more output derivative than would be considered in the centralized case; this simple modification surprisingly allows not only stabilization but effective pole placement, for minimumphase square-invertible uniform-rank decentralized plants. We note that the Special Coordinate Basis (SCB) for linear systems [11], [12] serves as a core tool for our design, because it fully exposes both the infinite- and finite-invariant zero structure of the plant, and hence permits us to properly use the derivatives of local observations in design. From the SCB, it becomes clear that minimum-phase uniformrank plants are amenable to high-gain decentralized control, analogously to the asymptotic time-scale and eigenstructure assignment (ATEA) design for centralized systems [11], [13]. The SCB also permits us to relate the decentralized fixed modes of a plant with its (central) invariant zeros, and guides us toward design for more general plants.

The second core aspect of our development is to obtain proper controller implementations of the conceptualized multi-derivative control scheme. That is, noting that derivatives of outputs cannot be exactly computed directly, we seek for proper controllers that approximate the derivative- controller dynamics in the closed loop. Such approximation of derivative feedback with proper controls turns out to be non-trivial in our context, specifically because derivatives up to and including the plant's relative degree are being used in feedback. Conceptually, these extra output derivatives contain not only state information but concurrent input information (possibly from several channels in the decentralized system), and so their approximation with proper controllers requires considerable care. Here, we demonstrate that proper finite-dimensional implementations, i.e. multiple lead-compensator implementations, can always be designed to match the performance of the derivative-based control and hence to achieve stabilization and pole placement for decentralized plants. Motivated by the common presence of delays in modern networks and the ease of implementing delay controllers in some settings, we also explore implementation of the derivative-based controllers using multipledelay compensators. In particular, we construct delay-based controllers that match the derivative control scheme's performance for a broad class of uniform-rank-1 plants. We are continuing to expand our understanding of delay-based and other implementation methods in our ongoing work, and here give some conjectures and pointers with regard to this further effort. We note that our efforts on proper implementation draw on classical literature on algorithms for solving linear systems [33], on retarded- and neutraltype delay systems [29], [31], and specifically on multipledelay controls [30]. Through the two-step design process, we are able to construct fully decentralized controllers with low complexity and distributed actuation effort.

Decentralized systems are often strongly impacted both by constraints on the agents and by network limitations and variations. An essential advantage of our delay-based control methodology is its effectiveness even in the presence of these harsh constraints/limitations. Specifically, actuator saturation nonlinearities are ubiquitous in both sensingagent network and infrastructural applications [19], [23]. While controller design under saturation has been extensively studied for centralized systems [27], design under saturation for decentralized systems is wholely unknown (see [32] for partial existence conditions). Also, network communications/sensing are routinely subject to delays, and so controlling networks with inherent delays is critically important. In the interest of space, we do not pursue design in the presence of constraints/delays here, but our complementary work [28] begins to address this case.

The remainder of the article is organized as follows. In Section 2, we motivate and introduce the decentralized control problem for uniform-rank plants. Section 3 develops the multiple-derivative control paradigm, and demonstrates stabilization and pole placement for minimum-phase uniform-rank decentralized plants using this methodology. Section 4 describes both lead-compensator and delay-based implementations of the multi-derivative controllers. Section 5 introduces one class of models, for infrastructure networks, that falls within the class of plants studied here; many other examples can be found in the extended document [14]. 


\section{Problem Formulation}

In this section, we motivate the uniform rank system models, and formulate the decentralized controller design problem for such systems.

Uniform rank models-those with the property that each infinite zero is of the same order-are of broad interest in control theory [9], and are much more general models than the sensing-agent network models commonly studied in the network control community, in that they can represent networks with internally-coupled agents/channels (i.e., ones for which the state matrix $A$ in Equation 1 is not block diagonal). Because of uniform rank models' structural generality, they can represent not only quite general autonomous-agent networks such as autonomous vehicle teams and sensor networks, but also various other modern hardwired/infrastructure networks, such as power networks, air traffic networks, and virus spreading networks [19]. For instance, in epidemic spreading and control applications, the typical multi-group SIR (Susceptible/Infected/Removed) and SEIR (Susceptible/Exposed/Infected/Removed) epidemic models are intrinsically uniform-rank 1 and 2 systems respectively.

Formally, in this paper, we consider stabilizing the general decentralized system

$$
\begin{aligned}
& \dot{\mathbf{x}}=A \mathbf{x}+\sum_{i=1}^{v} B_{i} \mathbf{u}_{\mathbf{i}} \\
& \mathbf{y}_{\mathbf{i}}=C_{i} \mathbf{x}
\end{aligned}
$$

where $\mathbf{x} \in R^{n}, v$ is the number of channels, $\mathbf{u}_{\mathbf{i}} \in R^{m_{i}}$, and $\mathbf{y}_{\mathbf{i}} \in R^{p_{i}}$, under the conditions that the centralized system is uniform rank [9], square invertible, minimum phase, and for each channel the number of inputs and outputs are equal, i.e. $m_{i}=p_{i}$ for all $i=1, \ldots, v$. For convenience, we define $\mathbf{u}=\left[\begin{array}{lll}\mathbf{u}_{1}^{T} & \ldots & \mathbf{u}_{v}^{T}\end{array}\right]^{T}, \mathbf{y}=\left[\begin{array}{lll}\mathbf{y}_{1}^{T} & \ldots & \mathbf{y}_{v}^{T}\end{array}\right]^{T}, B=$ $\left[\begin{array}{llll}B_{1} & B_{2} & \ldots & B_{v}\end{array}\right]$, and $C=\left[\begin{array}{llll}C_{1}^{T} & C_{2}^{T} & \ldots & C_{v}^{T}\end{array}\right]$. Then we can condense the system (Equation 1) into

$$
\begin{aligned}
& \dot{\mathbf{x}}=A \mathbf{x}+B \mathbf{u} \\
& \mathbf{y}=C \mathbf{x},
\end{aligned}
$$

where $B \in R^{n \times m}, C \in R^{m \times n}$, and the number of inputs/outputs is $m=\sum_{i=1}^{v} m_{i}$.

Our goal is to design a decentralized controller (i.e., each $\mathbf{u}_{i}$ only feeds back local output $\mathbf{y}_{i}$ ) to stabilize system (1). Specifically, we consider using multiple derivatives of $\mathbf{y}_{i}$ for stabilization, and also consider the controller's delay or lead compensator implementation.

The uniform-rank system model that we address here is much more general than the double-integrator networks analyzed in [1], [28]. We notice that uniform rank systems do not enjoy the special scaling properties of double-integrator models, and clearly require different tools for design. For us, they turn out to provide a stepping-stone toward decentralized controller design for general LTI plants, and permit exposition of the fundamentals underlying multiplederivative decentralized control.
The SCB proves a valuable tool in designing controllers for uniform rank systems, and in fact motivates study of uniform rank systems as a step toward design for general LTI decentralized systems. Conceptually, using the SCB form and applying a further state transformation, each channel's input in the decentralized uniform rank system can be viewed as driving (coupled) chains of integrators of the same length to produce the local observations. By taking derivatives of the local observations up to (one less than) the order of the integrator chain in the control law, the state dynamics associated with the entire infinite-zero structure becomes available to the channels together; hence, it is not altogether surprising that stabilization and even high performance design can be achieved provided the system is minimum phase. Just as for the double-integrator network, we find that taking one more derivative than is needed for centralized control (thus, up to the order of the integrator chain) yields local information that permits coordination of the channels in achieving control. We note that, from the SCB representation, the uniform rank system is peculiar in that local outputs can be viewed as integrations of inputs combinations upon state transformation (specifically because the depths of the integrator chains in the SCB are identical), and hence taking local derivatives serves to identify the entire state dynamics associated with the infinite-zero structure.

\section{Multiple-Derivative Controller Design For UNIFORM RANK SYSTEMS}

In this section, we present the design of multiple-derivative decentralized controllers for stabilization and effective pole placement, for square-invertible and minimum-phase uniform rank systems with the same number of inputs as outputs in each channel. The following theorem summarizes the stabilization result.

Theorem 1: Consider a general decentralized plant (Equation 1), that is uniform rank with rank $l$, square invertible, minimum phase, and for which $m_{i}=p_{i}$ for $i=$ $1, \ldots, v$. The system can be stabilized using linear feedback of the observation vector and its first $l$ derivatives at each channel.

\section{Proof:}

We shall show that the decentralized output feedback law

$$
\mathbf{u}=\alpha_{1} \mathbf{y}+\alpha_{2} \mathbf{y}^{(1)}+\ldots+\alpha_{l+1} \mathbf{y}^{(l)}
$$

can stabilize the plant. Specifically, we prove using the SCB that a high gain control of the above form can place the closed-loop eigenvalues into the OLHP (and in fact allow free assignment of all eigenvalues except those associated with the centralized invariant zero dynamics), assuming the plant is centrally minimum phase.

From the SCB [12], the system of interest (Equation 2) has $l m$ infinite zeros and $n-\operatorname{lm}$ (finite) invariant zeros. Denote that, in the SCB, the states associated with finite invariant zero dynamics are $\mathbf{x}_{\mathbf{a}}\left(\mathbf{x}_{\mathbf{a}} \in R^{n-l m}\right)$. From the development of the SCB [12] and a little algebra, it is immediate that there exists a state transformation $\Gamma$ such 
that $\Gamma \mathbf{x}=\left[\begin{array}{llll}\mathbf{x}_{\mathbf{a}}{ }^{T} & \mathbf{y}^{T} & \ldots & \mathbf{y}^{(l-1)^{T}}\end{array}\right]^{T}$. From the SCB, the invariant zero dynamics are known to take the form $\dot{\mathbf{x}}_{a}=$ $A_{a a} \mathbf{x}_{\mathbf{a}}+B_{0} \mathbf{y}$, where $A_{a a}$ and $B_{0}$ are matrices of appropriate dimensions. Also, a system with uniform rank $l$ has the property (by definition) that the Markov parameters $C A^{i} B$ are 0 for $i=0, \ldots, l-2$, and $C A^{l-1} B$ is nonsingular with rank $m$ [9]. Hence, we can easily see that $\mathbf{y}^{(l-1)}=C A^{l-1} \mathbf{x}$. Taking one more derivative leads to $\mathbf{y}^{(l)}=C A^{l} \mathbf{x}+C A^{l-1} B \mathbf{u}$.

Combining the above quantities, we obtain the state dynamics under the state transformation $\Gamma$ :

$\left[\begin{array}{c}\mathbf{x}_{\mathbf{a}} \\ \mathbf{y}^{(1)} \\ \vdots \\ \mathbf{y}^{(l-1)} \\ \mathbf{y}^{(l)}\end{array}\right]=\left[\begin{array}{cccccc}A_{a a} & B_{0} & 0 & 0 & \ldots & 0 \\ 0 & 0 & I & 0 & \ldots & 0 \\ 0 & 0 & 0 & I & \ldots & 0 \\ \vdots & \vdots & \vdots & \vdots & \vdots & \vdots \\ P_{1} & P_{2} & P_{3} & P_{4} & \ldots & P_{l+1}\end{array}\right]\left[\begin{array}{c}\mathbf{x}_{\mathbf{a}} \\ \mathbf{y} \\ \vdots \\ \mathbf{y}^{(l-2)} \\ \mathbf{y}^{(l-1)}\end{array}\right]+\left[\begin{array}{c}0 \\ 0 \\ \vdots \\ 0 \\ Q\end{array}\right] \mathbf{u}$

where the $P_{i}$ are matrices of appropriate dimension that satisfy $\left[\begin{array}{lll}P_{1} & \ldots & P_{l+1}\end{array}\right]=C A^{l}\left(\Gamma_{2} \Gamma_{1}\right)^{-1}$ and $Q=C A^{l-1} B$ is non-singular.

Now consider using the output feedback control law (3). The closed loop dynamics are

$$
\left[\begin{array}{c}
\mathbf{x}_{\mathbf{a}} \\
\mathbf{y}^{(1)} \\
\vdots \\
\mathbf{y}^{(l-1)} \\
\mathbf{y}^{(l)}
\end{array}\right]=\bar{A}\left[\begin{array}{c}
\mathbf{x}_{\mathbf{a}} \\
\mathbf{y} \\
\vdots \\
\mathbf{y}^{(l-2)} \\
\mathbf{y}^{(l-1)}
\end{array}\right]
$$

where $L=\left(I-\alpha_{l+1} Q\right)^{-1}$ and

$$
\bar{A}=\left[\begin{array}{ccccc}
A_{a a} & B_{0} & 0 & \ldots & 0 \\
0 & 0 & I & \ldots & 0 \\
0 & 0 & 0 & \ldots & 0 \\
\vdots & \vdots & \vdots & \vdots & \vdots \\
0 & 0 & 0 & \ldots & I \\
L P_{1} & L\left(P_{2}+\alpha_{1} Q\right) & L\left(P_{3}+\alpha_{2} Q\right) & \ldots & L\left(P_{l+1}+\alpha_{l} Q\right)
\end{array}\right.
$$

When high gains $\alpha_{1}, \ldots \alpha_{l+1}$ are used, $L\left(P_{i+1}+\alpha_{i} Q\right)$ approaches $-\frac{\alpha_{i}}{\alpha_{l+1}} I$. Furthermore, $L P_{1}$ becomes small, specifically of order $\frac{1}{\alpha_{l+1}}$, and hence is of lower order than $L\left(P_{i+1}+\right.$ $\left.\alpha_{i} Q\right)$ as long as $\alpha_{1}, \ldots \alpha_{l}$ are chosen proportional to $\alpha_{l+1}$. Hence, through a simple time-scale argument, we see that $n-l m$ poles converge to the invariant zeros (eigenvalues of $A_{a a}$ ), while the remaining poles can be placed at $l$ arbitrarilyselected locations through choice of $\alpha_{1}, \ldots, \alpha_{l-1}$. Thus, we have shown that stability can be achieved.

This theorem shows that a multiple-derivative controller can stabilize a square-invertible minimum-phase uniform rank system with the same number of inputs and outputs at each channel. In fact, identical gains for each channel are sufficient. This proof is based on the analysis of the system's SCB form, which exhibits the system's intrinsic integrator chain pathways between inputs and local outputs when an appropriate transformation is applied. It is easy to see that the proof naturally leads to a high-performance controller design, as formalized in the following Theorem
2. Specifically, for a minimum-phase uniform-rank system of rank $l$, we can design the multiple-derivative control to place the freely-assignable poles at desirable locations and the remaining pole at the (centralized) finite invariant zeros, using high gain control. The following algorithm can be used for design:

\section{Algorithm 1:}

- To place roots at locations $x_{1}, \ldots, x_{l}$ (that are closed under conjugation), find $k_{1}, \ldots, k_{l}$ such that the roots of $\lambda^{l}+k_{l} \lambda^{l-1}+\ldots+k_{1}=0$ are $x_{1}, \ldots, x_{l}$.

- Apply the following multiple-derivative control law:

u, (4)

$$
\mathbf{u}=k_{1} k_{l+1} \mathbf{y}+k_{2} k_{l+1} \mathbf{y}^{(1)}+\ldots+k_{l} \mathbf{y}^{(l-1)}+k_{l+1} \mathbf{y}^{(l)},
$$

where $k_{l+1}$ is chosen to be large.

The following theorem formalizes the performance of this design:

Theorem 2: $\quad$ Consider a general decentralized plant (Equation 1) which is centrally uniform rank with rank $l$, square invertible, minimum phase, and for which $m_{i}=p_{i}$ for $i=1, \ldots, v$, and say that we use the multiple derivative controller (Equation 6) generated by the Design Algorithm 1. By choosing $k_{l+1}$ sufficiently large, we can place $n-l m$ poles arbitrarily close to the (finite) invariant (centralized) zeros of the plant, and groups of $m$ poles near each desired location $x_{1}, \ldots, x_{l}$.

Proof: The proof of theorem 1 directly shows that by choosing $k_{1}, \ldots, k_{l}$ such that $x_{1}, \ldots, x_{l}$ are the roots of $\lambda^{l}+k_{l} \lambda^{l-1}+$ $\ldots+k_{1}=0$, and setting $k_{l+1}$ sufficiently large, the multiplederivative-based controller $\mathbf{u}=k_{1} k_{l+1} \mathbf{y}+k_{2} k_{l+1} \mathbf{y}^{(1)}+\ldots+$ $7_{l+1} \mathbf{y}^{(l)}$ is capable of placing group of $m$ poles arbitrarily close to locations $x_{1}, \ldots, x_{l}$, while the remaining $n-l m$ poles approach the fixed invariant zeros.

This theorem demonstrates a way to design a highperformance multiple-derivative controller. Note here that we have the freedom of placing group of poles arbitrarily close to $l$ locations, with each location attracting $m$ poles. This concept of group pole placement was elaborated in [1]. Briefly, group pole placement permits design of crucial system parameters such as the dominant eigenvalue or the dominant eigenvalue ratio, which are sufficient for almost all performance designs. Furthermore, through an inverse-optimality argument, high performance (optimality) with respect to a quadratic cost measure can be demonstrated.

Our method of design yields several further insights into the decentralized control of uniform rank systems. First, we note that the design using the SCB indicates a connection between a system's decentralized fixed modes [20] and centralized invariant zeros (e.g., [21]). Precisely, noting that our design can freely assign all closed-loop eigenvalues except those that approach the invariant zeros under high gain, we recover that the decentralized fixed modes are a subset of the centralized invariant zeros of the plant. Thus, we further obtain that centrally minimum-phase uniform-rank plants do not have any closed right-half-plane fixed modes. 
A second important observation from the design process is that derivatives of the local observations up to the "relative degree" of the plant (the common depth of the integrator chains) provide the channels with increasing information about the global state (upon feedback), and hence give increasing capability of control. Precisely, each derivative permits us to modify one block representing the couplings between the chains in the SCB (Equation 5), albeit in a decentralized way. The highest derivative (the one equal to the "relative degree") provides each channel with information regarding the actuations throughout the network, and thus turns out to permit the network to invert the couplings among the channels and so facilitate control using the lower derivatives, also see [1] for further discussion.

\section{LEAD-COMPENSATOR AND DELAY IMPLEMENTATION}

In Section 3, we introduced a promising paradigm for decentralized controller design for uniform-rank plants, that is based on feedback of output derivatives up to the relative degree of the plant at each channel. This philosophy for decentralized control is compelling in that it permits stabilization and pole placement while distributing complexity among the channels. However, we must stress that the derivative-based framework given in Section 2 is only a philosophy or paradigm for design: the obtained multiplederivative controllers are not proper, i.e. they require arbitrary high-frequency gain, and cannot be implemented directly unless the derivative signals are themselves measured. Thus, a second critical aspect of our design process is the approximation of the multiple-derivative conrollers for decentralized control with proper compensation schemes, through appropriate approximation of the derivative information used in feedback. These derivatives can be approximated in various ways, including using multiple-delay approximations (e.g., approximating $\dot{y}$ as $\frac{y(t)-y(t-\delta)}{\delta}$ ), using delayed measurements/approximations of the derivatives themselves (e.g. $\dot{y}(t-\delta)$ ), or using lead compensation, among others. Here, we shall study lead-compensation and multiple-delay approximations.

The philosophy of envisioning multiple-derivative-based controls and then using proper implementations is common in both classical and modern (centralized) control theory. Numerous means for proper implementation have been studied, including (finite-dimensional) lead compensation schemes and (infinite-dimensional) delay-based and delayapproximation controllers. These schemes can typically be made to approximate the derivative-based control arbitrarily well by making the controller's bandwidth sufficiently large. Thus, at first glance, it seems that proper implementation of the derivative-based decentralized control scheme may be routine. However, we call the reader's attention to a unique feature of our design, that derivatives equal to the (common) relative degree of the plant are used in each channel, or in other words that the channels directly use in feedback the concurrent inputs of multiple channels. An approximation of the feedback with a proper controller thus serves to replace this infeasible input feedback with a delayed or smoothed version, and so it is not surprising that some care is needed to develop and verify an approximation that replicates the derivative-based control. We note that some preliminary insights into such approximations can be found in the literature on delayed systems of neutral type [29], [30], but much remains to be done in designing and characterizing them.

Here, we detail progress on our ongoing effort to develop proper implementations for derivative-based decentralized controllers. In particular, we present a lead-compensator approximation that works in generality for uniform-rank decentralized plants, and hence yields a complete design for the class of models considered here. Motivated by the common presence of delays in networks and the natural use of delays in feedback in some applications, we also pursue multiple-delay approximations. Our preliminary efforts in this direction show that proper approximations can be obtained for a broad class of uniform-rank-1 plants. We leave it to future work to pursue more general delay implementations. We note that our studies of implementation here build on several previous efforts, including a lead-compensator implementation for stabilization and pole placement in double-integrator networks [28] and numerous foundational studies in implementation of multiple-derivative controllers using multiple-delay approximations or delayedderivative approximations [29]-[31].

Here in this section, we first consider the implementation of the multiple-derivative controller using lead compensators, for the uniform-rank system. We will show that this implementation maintains the stability of the closed-loop system by generating $n$ poles close to the locations of the poles using a multiple-derivative controller, while introducing $\frac{(1+l) l}{2} m$ poles far left in the complex plant. The proof of the implementation result immediately provides an explicit procedure for design. Here is the main result:

Theorem 3: Consider a general decentralized plant (Equation 1) which is centrally uniform rank with rank $l$, square invertible, minimum phase, and for which $m_{i}=p_{i}$ for $i=1, \ldots, v$, and consider any multiple-derivative controller (as designed per Equation 3) that stabilizes the plant. Consider a decentralized (observable, controllable) lead-compensator implementation of the derivative control that has the following transfer function: $u(s)=\alpha_{1} y(s)+$ $\alpha_{2}\left(I+\Lambda_{11} \varepsilon s\right)^{-1} s y(s)+\alpha_{3}\left(I+\Lambda_{21} \varepsilon s+\Lambda_{22} \varepsilon^{2} s^{2}\right)^{-1} s^{2} y(s) \ldots+$ $\alpha_{l+1}\left(I+\Lambda_{l 1} \varepsilon s+\Lambda_{l 2} \varepsilon^{2} s^{2}+\ldots \Lambda_{l l} \varepsilon^{l} s^{l}\right)^{-1} s^{l} y(s)$, where all $\Lambda_{i j}$ are full-rank diagonal matrices. By choosing $\varepsilon$ arbitrarily small, and choosing all $\Lambda_{i j}$ appropriately, the leadcompensator implementation of the control also stabilizes the plant. In fact, $n$ of the closed-loop poles approach those that would be obtained through the derivative-based control, while the remaining can be moved arbitrarily far left in the complex plane.

Proof: We prove the result by analyzing the pole locations of the closed-loop dynamics using the multi-lead compensator for control. The proof closely follows the proof for multilead-compensator implementation for the double-integrator 
integrator network that we have given in [28], thus our presentation here is terse and we refer the reader to [28] for further details.

For convenience, let us denote $L_{1}(s)=I+\Lambda_{11} \varepsilon s, L_{2}(s)=$ $I+\Lambda_{21} \varepsilon s+\Lambda_{22} \varepsilon^{2} s^{2}, \ldots, \quad L_{l}(s)=I+\Lambda_{l 1} \varepsilon s+\Lambda_{l 2} \varepsilon^{2} s^{2}+$ $\ldots \Lambda_{l l} \varepsilon^{l} s^{l}$. We then write down the dynamics of the closedloop plant by appending the controller dynamics to the plant dynamics shown in Equation 4, and hence obtain the closed-loop characteristic polynomial. Doing just a little bit of algebraic simplification, we obtain that the closed-loop characteristic polynomial is the determinant of the matrix

$$
C(s)=\left[\begin{array}{ll}
C_{11}(s) & C_{12}(s) \\
C_{21}(s) & C_{22}(s)
\end{array}\right]
$$

where $\quad C_{11}(s)=s I-A_{a a}, C_{12}(s)=B_{0} \prod_{i=1}^{l} L_{i}(s), \quad C_{21}(s)=$ $P_{1}$, and $C_{22}(s)=s^{l} I-\left(P_{2}+P_{3} s+\ldots+P_{l+1} s^{l-1}\right) \prod_{i=1}^{l} L_{i}(s)-$ $Q\left(\alpha_{1} \prod_{i=1}^{l} L_{i}(s)+\alpha_{2} s \prod_{i=2}^{l} L_{i}(s)+\ldots+\alpha_{3} s^{2} \prod_{i=1, i \neq 2}^{l} L_{i}(s)+\ldots\right.$ $\left.+\alpha_{l+1} s^{l} \prod_{i=1}^{l-1} L_{i}(s)\right)$.

The poles of the closed-loop system are the values $s$ that make $C(s)$ lose rank. Consideration of $C(s)$ clearly tells us that the closed-loop system has $n+\frac{(1+l) l}{2} m$ poles. As $\varepsilon$ approaches 0 , each $L_{i}$ approaches to $I$ for all $s$ in a ball of arbitrary-large size around the origin. Hence, we can see that the characteristic polynomial approaches to the one when the derivative controller is used in such a ball, through equivalencing this characteristic polynomial with the one obtained upon derivative control (Equation 5). Hence, $n$ poles approach the poles of the closed-loop system upon use the derivative controller, while the remaining poles become arbitrarily large in magnitude, as $\varepsilon$ is decreased toward 0 .

Now let us consider the poles that are far away from the origin in the complex plane: we will design the compensator parameters $\Lambda_{i j}$ to put these poles on the OLHP. To simplify this design, let us introduce the scaling $\bar{s}=\varepsilon s$ and also use the notation $\bar{L}_{i}(\bar{s})=I+\Lambda_{i 1} \bar{s}+\Lambda_{i 2} \bar{s}^{2}+\ldots \Lambda_{i i} \bar{s}^{i}$, for all $i$. With just a little algebra, we find that the closed-loop characteristic polynomial in this fast time-scale is the determinant of the following matrix:

$$
\bar{C}(\bar{s})=\varepsilon^{n} C(\bar{s})=\bar{s}^{n} \prod_{i=1}^{l} \bar{L}_{i}(\bar{s})-\bar{s}^{n} \alpha_{l+1} Q \prod_{i=1}^{l-1} \bar{L}_{i}(\bar{s})+\operatorname{Poly}(\varepsilon, \bar{s}),
$$

where $\operatorname{Poly}(\varepsilon, \bar{s})$ is a polynomial matrix in $\bar{s}$ with each entry of order at most $\varepsilon$, and of sufficiently low degree that the leading term of the characteristic polynomial does not change. From Equation 8, as $\varepsilon$ becomes small, $n$ poles approach the origin (in $\bar{s}), \frac{(l-1) l}{2} m$ poles approach values $\bar{s}$ such that $\bar{L}_{i}(\bar{s})$ loses rank for $i=1, \ldots, l-1$, and the remaining Im poles approach the values $\bar{s}$ such that the polynomial $\bar{L}_{l}(\bar{s})-\alpha_{l+1} Q$ loses rank. The $n$ poles approaching the origin in $\bar{s}$ correspond to the small-magnitude poles in the $s$ coordinates and are known to be in the OLHP.

Now let us choose all the parameters in the lead compensators, so to place all the additionally introduced $\frac{(1+l) l}{2} m$ poles in the OLHP. To do so, we notice that for each $i m$ poles that correspond to $\bar{L}_{i}(\bar{s})$ losing rank for $i=1, \ldots, l-1$, we can easily place them in the OLHP that by choosing each entry in the diagonal matrix of $\Lambda_{i j}$ positive and real, and having them satisfy the Routh Criterion entry-wise.

Finally let us consider the values $\bar{s}$ such that the polynomial matrix $\bar{L}_{l}(\bar{s})-\alpha_{l+1} Q=I-\alpha_{l+1} Q+\Lambda_{l 1} \bar{s}+\Lambda_{l 2} \bar{s}^{2}+$ $\ldots+\Lambda_{l l} \vec{s}^{l}$ loses rank. Assuming $\Lambda_{l l}$ is full rank, these poles are exactly the values $\bar{s}$ such that the polynomial matrix $\bar{s}^{l}+\Lambda_{l l}^{-1} \Lambda_{l(l-1)} \bar{s}^{\vec{s}-1}+\ldots+\Lambda_{l l}^{-1} \Lambda_{l 1} \bar{s}^{1}+\Lambda_{l l}^{-1}\left(I-\alpha_{l+1} Q\right)$ loses rank. When $\alpha_{l+1}$ is large, all principal minors of $I-\alpha_{l+1} Q$ are full rank (see [28] for details), and hence we can sequentially design the entries of the diagonal matrix $\Lambda_{l l}$ to make all the eigenvalues of $\Lambda_{l l}^{-1}\left(I-\alpha_{l+1} Q\right)$ real and positive [28], [33]. After $\Lambda_{l l}$ is determined, we then design $\Lambda_{l i}$ for $i=1, . ., l-1$. Specifically, using eigenvalue decomposition, we can easily choose appropriate $\Lambda_{l i}$ to place all $l m$ poles in the OLHP, see [28] for the details.

We have thus designed a decentralized multi-leadcompensation scheme that stabilizes the broad class of minimum-phase uniform-rank plants. We again stress that this design has two core aspects, a multi-derivative feedback conceptualization for stabilization and pole-placement, followed by a proper implementation of the controller concept. Let us also note again that the complexity in implementation stems from the need to use derivatives equal to the relative degree of the plant in feedback; this complexity is evidenced in the need for sequential design of the diagonal (filtering) matrix $\Lambda_{l l}$, so as to adequately replicate the highest-derivative feedback through the input transformation defined by the matrix $Q$. Interestingly, the implementation may in general require use of open-loop unstable controllers at some channels.

As an interesting alternative to the multi-leadcompensation implemenation, we are also developing delayfeedback-based implementations of the multi-derivative feedback. That is, we are considering approximation of an output derivative used in feedback, say $\dot{y}(t)$, as a first-difference, namely $\frac{y(t)-y(t-\varepsilon)}{\varepsilon}$. Such delaybased implementations are compelling for some network applications, because of the common presence of intrinsic delays and/or the ease of using stored output data in feedback. However, because delay-feedback yields infinitedimensional closed-loop dynamics, verification of stability and performance becomes much more intricate in this case, and so we are just beginning to obtain delay-based implementations for the multiple-derivative controllers. Here, let us specify a first result on delay-based control, namely regarding a broad class of uniform-rank-1 plants:

Theorem 4: Consider a decentralized plant (Equation 1) which is centrally uniform rank-1, square invertible, minimum phase, and for which $m_{i}=p_{i}$ for $i=1, \ldots, v$. Also assume that the input-transformation matrix $Q$ in the special coordinate basis representation of the plant (Equation 4) has the following property: there exists a commensurate block-diagonal matrix $D$ (one with $v$ blocks, of dimension $\left.m_{i} \times m_{i}\right)$ such that $Q D$ has positive real eigenvalues. Then 
a decentralized feedback controller of the form $\mathbf{u}=\gamma_{1} D \mathbf{y}+$ $\gamma_{2} D \mathbf{y}(t-\varepsilon)$ can be designed to stabilize the plant. In fact, such a controller can be designed to place $n$ closed-loop poles near a desired location on the negative real axis, while the remaining poles can be moved arbitrarily far left in the complex plane by making $\varepsilon$ small.

\section{Proof:}

From the development in Section 3, a decentralized derivative-feedback controller of the form $\mathbf{u}=\alpha_{1} D \mathbf{y}+\alpha_{2} D \dot{\mathbf{y}}$ with $\alpha_{1}<0$ and $\alpha_{2}<0$ can be designed to stabilize the uniform-rank-1 plant considered here, and in fact to place the $n$ closed-loop poles arbitrarily near to a desired location on the negative real axis. Let us consider approximating this feedback control using the proper decentralized compensator $\mathbf{u}=\alpha_{1} D \mathbf{y}+\alpha_{2} D(\mathbf{y}(t)-\mathbf{y}(t-\varepsilon))$. Notice that this compensator is of the form given in the theorem statement, with $\gamma_{1}=\alpha_{1}+\alpha_{2}$ and $\gamma_{2}=-\alpha_{2}$.

When the delay compensator is used, it can be shown with a little algebra that the closed-loop poles are the values $s$ such that the determinant of the following matrix (or characteristic polynomial) is nil, i.e. the matrix loses rank:

$$
\left[\begin{array}{cc}
s I-A_{a a} & -B_{0} \\
-P_{1} & s I-P_{2}-\alpha_{1} Q D-\alpha_{2} \frac{1-e^{-\varepsilon s}}{\varepsilon} Q D
\end{array}\right],
$$

where $A_{a a}, B_{0}, P_{1}$, and $P_{2}$ come from the SCB representation of the plant. We claim that, as $\varepsilon$ is made small, $n$ closedloop poles approach the poles when the derivative controller is used while the remaining (infinite number of) poles move arbitrarily far left in the complex plane.

To prove this, we note that $\frac{1-e^{-\varepsilon s}}{\varepsilon}$ can be made to approximate the derivative function $s$ arbitrarily well in any closed and bounded ball around the origin. Using this observation and equivalencing the characteristic polynomials upon delay and derivative control, we immediately see that $n$ poles approach the poles when the derivative controller is used as $\varepsilon$ is made small. Meanwhile, the remaining poles are seen to be outside this ball or arbitrary size, i.e. they can be made arbitrarily large in magnitude by making $\varepsilon$ small. It remains to show that these large eigenvalues are in the OLHP.

To characterize the large eigenvalues, let us apply the scaling $\bar{s}=\varepsilon s$. Substituting this scaling into the characteristic polynomial, eliminating order- $\varepsilon$ terms, and simplying the expression, we obtain that the poles (in the $\bar{s}$ domain) are the values $\bar{s}$ such that $\bar{s} I-\alpha_{2}\left(1-e^{-\bar{s}}\right) Q D$ loses rank. Doing an eigenvalue decomposition of $Q D$, we thus find that the poles are the roots of the expressions $\bar{s}-\alpha_{2}\left(1-e^{-\bar{s}}\right) \mu_{i}$, $i=1, \ldots, m$, where the $\mu_{i}$ are the eigenvalues of $Q D$ and are in the ORHP. However, from the proof of Theorem 2 in [30], it immediately follows that that the poles are in the OLHP, and in fact can be placed arbitrarily far to the left in the $s$ domain by making $\varepsilon$ sufficiently small.

\section{Example: An Infrastructure-Network Model}

Decentralized controller designs are needed for numerous modern infrastructures, such as for stopping virus spreads in human populations, reducing delays in air traffic networks, and achieving wide-area control in electric power systems. These infrastructure-network control problems differ from sensing-agent-network design problems, in that the network components' states evolve through interaction even without application of feedback: for instance, virus prevalences naturally evolve through inter-personal interactions, aircraft flows necessarily alter regional traffic counts, and generator phases in the power system align. A primary focus of our group's research has been to address design/control problems for several infrastructure networks [4], [19], [24]. Many infrastructure-network control and design problems that we have encountered can be viewed as decentralized controller design problems for uniform-rank plants, and hence the design methodology develop here can directly be applied. As a preliminary illustration of controller design for infrastructure networks, let us introduce a simplistic infrastructure network model that nevertheless captures certain traffic-flow and virus-spread dynamics [4], [19].

The simple infrastructure-network model that we consider comprises $n$ components, labeled $1, \ldots, n$. Each component has a scalar state $x_{i}(t)$, that we assume evolves ab initio through interaction with neighboring components, according to a linear differential equation. Furthermore, $v$ of the components $(1, \ldots, v$, WLOG) are amenable to feedback control: specifically, each of these components has available a scalar output $y_{i}(t)$ equal to the local state $x_{i}(t)$, which can be used to design an input signal $u_{i}(t)$ that actuates the local state dynamics. Precisely, the infrastructure network model that we consider is a $v$-channel decentralized plant with the following dynamics:

$$
\begin{array}{r}
\dot{\mathbf{x}}=\left[\begin{array}{ll}
G_{11} & G_{12} \\
G_{21} & G_{22}
\end{array}\right] \mathbf{x}+\left[\begin{array}{c}
I_{v} \\
0
\end{array}\right] \mathbf{u} \\
\mathbf{y}=\left[\begin{array}{ll}
I_{v} & 0
\end{array}\right] \mathbf{x},
\end{array}
$$

where $\quad \mathbf{x}=\left[\begin{array}{lll}x_{1} & \ldots & x_{n}\end{array}\right]^{T}, \quad \mathbf{u}=\left[\begin{array}{lll}u_{1} & \ldots & u_{v}\end{array}\right]^{T}, \quad \mathbf{y}=$ $\left[\begin{array}{lll}y_{1} & \ldots & y_{v}\end{array}\right]^{T}$, and the matrix $G_{11}$ has dimension $v \times v$. This very simple model with "hardwired" interactions encompasses representative dynamics in a couple infrastructural applications, including in virus-spread control and air traffic flow management, see [19], [24]. We also kindly ask the reader to see our ongoing work (e.g., [35]) for more complicated infrastructure network models, including ones with higher-order component dynamics, constraints/variations, and uncertainties; in our future work, we plan to address controller design in these more-general models by applying and enhancing the methodology introduced here.

The simple infrastructure-network model described above can straightforwardly be shown to be a uniform-rank-1 plant, with its $n-v$ zeros equal to the eigenvalues of the matrix $G_{22}$. Thus, from the results in Section 3 and 4, we immediately obtain that decentralized stabilization of the infrastructure network model can be achieved using either a multi-leadcompensator (with 1 memory element at each channel) or a multiple-delay controller, as long as $G_{22}$ has eigenvalues in the OLHP. Furthermore, for either implementation, the closed-loop poles can be placed in the complex plane as 
follows: $v$ poles can be placed near any single desirable location on the negative real axis, while $n-v$ poles are moved closed to the eigenvalues of $G_{22}$ and the remaining poles are driven abitrarily far left in the complex plane. Thus, stabilization and effective pole placement can be achieved for many infrastructure network models of the above form.

\section{Affiliations and Acknowledgements}

This work was partially supported by the National Science Foundation under Grants ECS-0528882, ECS-0725589, and ECS-0901137, and by the National Aeronautics and Space Administration under Grant NNA06CN26A. The first author is with the Department of Electrical Engineering, University of North Texas. The second and third authors are with the School of Electrical Engineering and Computer Science, Washington State University. The fourth author is with Department of Mathematics, University of Twente.

\section{REFERENCES}

[1] Y. Wan, S. Roy, A. Saberi, and A. Stoorvogel, "A multiplederivative and multiple-delay paradigm for decentralized control: introduction using the canonical double-integrator network," in Proceedings of AIAA 2008 Guidance, Navigation, and Control Conference, Honolulu, Hawaii, 18-21 August, 2008.

[2] D. Siljak, Decentralized Control of Complex Systems, Academic Press: Boston, 1994.

[3] J. P. Corfmat and A. S. Morse, "Decentralized control of linear multivariable systems," Automatica, vol. 12, pp. 479495, 1976.

[4] Y. Wan and S. Roy, "A scalable methodology for evaluating and designing air traffic flow management strategies under uncertainty," IEEE Transactions on Intelligent Transportation Systems, vol. 9, no. 4. pp. 644-656, 2008.

[5] C. L. DeMarco, "Control structures for competitive marketdriven power systems," Proceedings of the 40th IEEE Conference on Decision and Control, Orlando FL, Dec. 2001.

[6] S. Roy and A. Saberi,"Scaling: a Canonical design problem for networks," International Journal of Control, vol. 80, no. 8, pp. 1342-1353, August 2007.

[7] B. D. O. Anderson and J. B. Moore, "Time-varying feedback laws for decentralized control," IEEE Transactions on Automatic Control, Vol. 26, No. 5, pp. 1133-1139, October 1981.

[8] J. P. Corfmat and A. S. Morse, "Stabilization with decentralized feedback control," IEEE Transactions on Automatic Control, Vol. 21, No. 1, Feb. 1976. pp. 679-682.

[9] A. Saberi, "Simultaneous stabilization with almost disturbance decoupling part 1: uniform rank systems," in Proceedings of 24th Conference on Decision and Control, Ft. Lauderdale, FL, December, 1985.

[10] A. Saberi and P. V. Kokotovic and H. J. Sussmann, "Global Stabilization of Partially Linear Composite Systems," SIAM Journal on Control and Optimization, Vol. 28, No. 6, pp. 1491-1503, November 1990.

[11] A. Saberi, B. M. Chen, and P. Sannuti, Loop Transfer Recovery: Analysis and Design, Springer-Verlag, 1993.

[12] P. Sannuti and A. Saberi, "Special coordinate basis for multivariable linear system-finite and infinite zero structure, squaring down and decoupling," International Journal of Control, vol. 45, No. 5, pp. 1655-1704, 1987.

[13] S. Roy, A. Saberi, and Y. Wan, "On multiple-delay output feedback stabilization of LTI plants," International Journal of Robust and Nonlinear Control, in press.

[14] Y. Wan, S. Roy, A. Saberi, and A. Stoorvogel, "A MultipleDerivative and Multiple-Delay Paradigm for Decentralized Controller Design: Uniform-Rank Systems," (extended version), submitted to Dynamics of Continuous, Discrete and Impulsive Systems, for a Special Issue to be published in honor of Hassan Khalil's 60th birthday.
[15] R. M. Murray, K. J. Astrom, S. P. Boyd, R. W. Brockett, and G. Stein, "Future directions in control in an information-rich world," IEEE Control Systems Magazine, pp. 20-33, April 2003.

[16] J. Baillieul and P. J. Antsaklis, "Control and communication challenges in networked real-time systems," Proceedings of the IEEE, vol. 95, no. 1, pp. 9-28, Jan. 2007.

[17] S. Roy, A. Saberi, and A. Stoorvogel, "Toward a control theory for networks," editorial in the Special Issue on Communicating-Agent Networks, International Journal of Robust and Nonlinear Control, vol. 17, no. 10-11, p. 897, July 2007, edited by Sandip Roy, Ali Saberi, and Anton Stoorvogel.

[18] W. Ren, R. W. Beard, and E. M. Atkins, "Information consensus in multivehicle cooperative control," IEEE Control Systems Magazine, vol. 27, no. 2, pp. 71-82, 2007.

[19] Y. Wan, S. Roy, and A. Saberi, "Designing spatiallyheterogeneous strategies for control of virus spread," IET Systems Biology, vol. 2, No. 4, pp. 184-201, 2008.

[20] S. Wang and E. J. Davison, "On the stabilization of decentralized control system," IEEE Transactions on Automatic Control, vol. AC-18, pp. 473-478, October 1973.

[21] C. B. Schrader and M. K. Stein, "Research on system zeros: a survey," International Journal of Control, Vol 50, Number 4, pp. 1407-1433, 1989.

[22] J. K. Hale and S. M. V. Lunel, Introduction to Functional Difference Equations, Epring-Verlag, 1993.

[23] S. Roy, A. Saberi, and K. Herlugson, "Formation and alignment of distributed sensing agents with double-integrator dynamics," IEEE Press Monograph on Sensor Network Operations, April 2006.

[24] Y. Wan, S. Roy, and A. Saberi, "A new focus in the science of networks: toward methods for design," Journal of the Royal Philosophical Society, vol. 464, pp. 513-535, Mar. 2008.

[25] Yan Wan, Sandip Roy, Xu Wang, Ali Saberi, Tao Yang, Mengran Xue, Babak Malek, "On the structure of graph edge designs that optimize the algebraic connectivity," in Proceedings of the 47th IEEE Conference on Decision and Control, Cancun, Mexico, Dec. 2008.

[26] S. Roy, Y. Wan, and A. Saberi, "On time-scale designs for networks," International Journal of Control, vol. 82, no. 7, pp. 1313-1325, July 2009.

[27] A. Saberi, A.Stoorvogel, and P. Sannuti, Output Regulation and Control Problems with Regulation Constraints, SpringerVerlag: New York, 1999.

[28] Y. Wan, S. Roy, A. Saberi, and A. Stoorvogel, "The design of multi-lead-compensators for stabilization and pole placement in double-integrator networks under satuation," in Proceedings of 2009 American Control Conference.

[29] A. Stoorvogel, S. Roy, Y. Wan, and A. Saberi, "A class of neutral-type delay differential equations that are effectively retarded," to appear in the IEEE Transactions on Automatic Control.

[30] Y. Wan, S. Roy, A. Stoorvogel, and A. Saberi, "On multipledelay approximations of multiple-derivative controllers," in Proceedings of 2009 European Control Conference.

[31] S. Roy, A. Saberi, and Y. Wan, "On multiple-delay static output feedback stabilization of LTI plants," International Journal of Robust and Nonlinear Control, in press.

[32] A. Stoorvogel, A. Saberi, C. Deliu, and P. Sannuti, "Decentralized stabilization of time-invariant systems subject to actuator saturation," Advanced Strategies in Control Systems with Input and Output Constraints, LNCIS series (S. Tarbouriech et al eds.), Springer-Verlag, June 2006.

[33] M. E. Fisher and A. T. Fuller,"On the Stability of matrices and the convergence of linear iterative processes," Proceedings of the Cambridge Philosophical Society, vol. 45, pp. 417-425, 1958.

[34] Y. Wan, S. Roy, and A. Saberi, "A pre- + post- + feedforward compensator design for zero placement," submitted to International Journal of Control.

[35] S. Roy, Y. Wan, and A. Saberi, "A network control theory approach to virus-spread mitigation," in Proceedings of the 2009 IEEE International Conference on Technologies for Homeland Security, Boston MA, May 2009. 